\title{
Transtorno do espectro autista: descobertas, perspectivas e Autism Plus
}

\author{
Autism spectrum disorder: findings, perspectives and Autism Plus.
}

\author{
Vinícius Magalhães Borges ${ }^{1}$, Lília Maria Azevedo Moreira2* \\ ${ }^{1}$ Graduação em Ciências Biológicas pela UFBA. Mestre em Genética pelo PPGGENBIO, UFBA, Bahia; ${ }^{2}$ Mestre e \\ Doutora em Genética pela USP, Professora Titular de Genética, UFBA, Bahia
}

\begin{abstract}
Resumo
Introdução: o Transtorno do Espectro Autista (TEA) é um distúrbio complexo do desenvolvimento sendo alvo de estudos desde 1943, quando o primeiro caso foi descrito. Objetivo: $O$ presente trabalho objetivou revisar as informações mais relevantes das últimas décadas a respeito do Transtorno do Espectro Autista, construindo bases científicas para o Autism Plus de Gilberg. Metodologia: foi realizada revisão da literatura nas bases de dados CAPES/MEC e via PUBMED nos idiomas português e inglês de janeiro de 1978 a janeiro de 2017. Resultados: após a aplicação dos critérios de seleção e exclusão, 46 artigos foram elegíveis para a análise com foco em áreas como: Histórico do autismo; Evolução do Diagnostic and Statistical Manual of Mental Desorders (DSM); Autismo idiopático e secundário; Aspectos epidemiológicos; Distribuição de TEA por sexo; Bases neurobiológicas; Bases genéticas; Autism Plus. Tanto os critérios de autismo quanto a sua classificação sofreram mudanças ao longo das décadas, bem como o número de genes sabidamente associados e candidatos a condição. Foram descobertos fatores ambientais determinantes, como uso de abortivos durante a gestação, e foi possível estabelecer a prevalência desproporcional de ocorrência de TEA no sexo, além de diversas condições psiquiátricas e genéticas em comorbidade. Conclusão: o TEA é uma condição complexa, com fenótipo amplo, e vasta condições passíveis de serem encontradas em comorbidade, quadro denominado Autism Plus. A ocorrência de Autism Plus síndromes genéticas é frequentemente observada, e pode compartilhar etiologia comum, por pleiotropia.
\end{abstract}

Palavras-chave: Transtorno do Espectro Autista. Transtorno Autístico.

\begin{abstract}
Introduction: autistic Spectrum Disorder (ASD) is a complex developmental disorder being studied since 1943, when the first case was described. Objective: This paper aimed to review the most relevant information of the last decades regarding Autism Spectrum Disorder, building scientific basis for Gilberg Autism Plus. Methodology: we performed a literature review in CAPES/MEC databases and via PUBMED in Portuguese and English languages from January 1978 to January 2017. Results: after applying the selection and exclusion criteria, 46 articles were eligible for the analysis with focus on areas such as: Autism history; Evolution of the Diagnostic and Statistical Manual of Mental Disorders (DSM); Idiopathic and secondary autism; Epidemiological aspects; Distribution of ASD by sex; Neurobiological bases; Genetic bases; Autism Plus. Both the autism criteria and their classification have undergone changes over the decades, as well as the number of genes known to be associated and candidates for the condition. Was found determinant environmental factors, such as abortive use during gestation, and was possible to establish the disproportionate prevalence of occurrence of ASD by sex, in addition to establish several psychiatric and genetic conditions in comorbidity. Conclusion: TEA is a complex condition, with broad phenotype, and vast conditions that can be found in comorbidity, a frame called Autism Plus. The occurrence of Autism Plus genetic syndromes is frequently observed, and may share common etiology, by pleiotropy.
\end{abstract}

Keywords: Autism Spectrum Disorder. Autistic Disorder.

\section{INTRODUÇÃO}

O Transtorno do Espectro Autista (TEA) é reconhecido como um distúrbio complexo do desenvolvimento, caracterizado por anormalidades abrangentes em três domínios do desenvolvimento: (1) interação social recíproca, (2) comunicação e (3) presença de um repertório comportamental de interesses restritos, repetitivo e estereotipado, sendo estas anormalidades verificadas logo nos primeiros meses de vida. O TEA compreende uma condição clinicamente heterogênea a qual envolve uma faixa de comportamentos onde o autismo se insere,

Correspondente/ Corresponding: *Lília Maria de Azevedo Moreira - End: Rua Barão de Geremoabo, 147 Ondina. - Salvador - Ba. CEP : 40170-290 - Tel: (71) 98197-6112 - E-mail: lazevedo@ufba.br embora o TEA unificado como uma única condição tenha sido pactuado apenas em 2013. O fenótipo autista é muito variável e verificam-se tanto autistas clássicos, com ausência de comunicação verbal e deficiência intelectual grave, como também autistas com habilidades verbais e inteligência normal, mas com sociabilidade comprometida (CARVALHEIRA; VERGANI; BRUNONI, 2004). As causas do autismo são ainda pouco compreendidas e os avanços metodológicos na pesquisa genética têm trazido novas informações sobre a etiologia do distúrbio.

O termo "autismo" foi primeiramente usado por Eugene Bleuler, em 1911, para designar crianças que, aparentemente, haviam perdido contato com a realidade e apresentavam dificuldade de comunicação. De acordo com Baker (2013), o psicanalista Leo Kanner foi quem 
de fato descreveu, em 1943, o autismo como "Distúrbio Autístico de Contato Afetivo". O seu trabalho foi realizado através da descrição de 11 crianças com características como "solidão extremamente autística", ecolalia, ansiedade obsessiva e busca de manutenção da rotina.

O autismo é, desde a descrição do primeiro caso, interesse de estudo da psiquiatria, sendo por muitos anos visto como a apresentação inicial de uma doença mais severa, a esquizofrenia infantil. De acordo com Assumpção e Pimentel (2000), o próprio Kanner acreditava na associação entre autismo e esquizofrenia. Só em 1970 foi legitimada a origem biológica do autismo e aceita a possibilidade da condição ocorrer - em alguns casos - em associação com deficiência intelectual, antes teoria inaceitável.

Ao longo das últimas décadas muito do que se sabia sobre o autismo foi revisto, ao passo que progressos foram feitos - decorrentes do desenvolvimento da medicina, especialmente, e de áreas de estudo como a genética, psicologia e psiquiatria - gerando um volume amplo de dados a respeito do tema.

O objetivo do presente trabalho é revisar as informações mais relevantes das últimas décadas a respeito do Transtorno do Espectro Autista, construindo bases científicas para o Autism Plus de Gilberg.

\section{METODOLOGIA}

Para elaboração desta revisão foram selecionados artigos com temas específicos a respeito do TEA de forma a, aqui reunidos, fornecerem informações amplas e atualizadas sobre o tema, sobretudo do ponto de vista da associação com a genética.

Sistematicamente, foram definidas as bases de dados pesquisadas, Periódicos CAPES/MEC e via PUBMED, com corte temporal de janeiro de 1978 até janeiro de 2017. As palavras-chave foram pesquisadas empregando-se o inglês e o português como idiomas.

Para a seleção de artigos, foram utilizados os seguintes critérios: 1 - artigo científico original ou revisão, publicado em periódicos nacionais e internacionais relacionados ao Transtorno do Espectro Autista (TEA); 2 - artigos nos idiomas português ou inglês; 3 - artigos relacionados ao tema TEA; 4 - artigos relacionados ao tema Síndromes Genéticas; 5 - artigos relacionados ao tema Histórico do Autismo; 6 - Artigos relacionados ao tema Evolução do Diagnostic and Statistical Manual of Mental Desorders (DSM); 7 - Artigos relacionados ao tema Autismo idiopático e secundário; 8 - Artigos relacionados ao tema Aspectos epidemiológicos; 9 - Artigos relacionados ao tema Distribuição de TEA por sexo; 10 - Artigos relacionados ao tema Bases neurobiológicas; 11 - Artigos relacionados ao tema Bases genéticas; e 12 - Artigos relacionados ao tema Autism Plus. Ainda foram usados os descritores: Autistic Disorder, Diagnostic and Statistical Manual of Mental Disorders e Autism Spectrum Disorder. Como também foram utilizados os qualificado- res: Epidemiology (EP), Etiology (ET), History (HI), Genetics (GE), Pathology (PA) e Psychology (PX). Foram excluídos estudos: 1 - resumo com acesso restrito; 2 - resumo com acesso disponível e artigo restrito; 3 - com publicação em outros idiomas. Comentários, editoriais, cartas, artigos completos e artigos de resumo que não apresentaram TEA como tema principal foram excluídos.

\section{RESULTADOS E DISCUSSÃO}

Após a busca bibliográfica, foram selecionados 46 artigos condizentes com os critérios metodológicos estabelecidos.

\section{Histórico do Diagnostic and Statistical Manual of Mental Desorders}

De acordo com Baker (2013), em 1980 surgiu o Diagnostic and Statistical Manual of Mental Desorders III, o DSM III. De importância imensurável, este foi o DSM que mais se destacou, definindo o autismo infantil como uma desordem de desenvolvimento, assim o distanciando de esquizofrenia. $\mathrm{O}$ autismo então foi classificado como abrangente em três domínios: falha de interação e resposta social, prejuízo em habilidades de comunicação e repostas inadequadas a vários aspectos do meio, sendo tudo inicialmente observado a partir dos 30 primeiros meses de vida.

Em 1987 foi lançado o DSM III-R, agregando uma definição mais complexa da desordem autística e apresentando uma série de 16 critérios relacionados aos 3 domínios, dos quais 8 critérios são requeridos para a classificação do indivíduo com desordem autística. Foi ainda no DSM III-R que foi apresentado um termo específico para indivíduos que apresentavam alguns, mas não todos os sintomas da desordem, "Pervasive Developmental Disorder, Not Otherwise Specified (PDD-NOS)".

O DSM-IV (APA, 1994) só foi lançado em 1994, enquanto o DSM IV-TR foi elaborado em 2000, ambos trazendo um refinamento nos critérios de diagnóstico. Foram nestes DSMs que o número de desordens de desenvolvimento foi ampliado para 5, incluindo Asperger, Síndrome de Rett e Transtorno Desintegrativo da Infância. Embora a Síndrome de Rett e o Transtorno Desintegrativo da Infância estejam inclusos na listagem do DSM-IV, eles não são de fato considerados TEA, mas devem ser avaliados no diagnóstico de cada criança por compartilharem sinais na sintomatologia clínica. A Síndrome de Rett tem etiologia genética conhecida - mutações no gene $\mathrm{MeCP}_{2^{\prime}}$ e o Transtorno Desintegrativo da Infância está associado a transtornos metabólicos e neurológicos.

Em 2013 foi publicado o DSM-V (APA, 2013), com alterações drásticas nos critérios de diagnóstico da TEA, trazendo uma alteração de 3 para 2 domínios afetados, sendo estes: 1 - Inadequação em reciprocidade social e comunicativa e prejuízo na interação social; 2 - Padrões comportamentais restritos e repetitivos. Neste DSM, Asperger foi removido das desordens, bem como o PDD- 
-NOS e o autismo clássico, gerando uma unificação de termos onde a totalidade de quadros fenotípicos passa a ser referida como espectro autista, ou TEA (Transtorno do Espectro Autista).

\section{Fatores Ambientais Associados ao TEA Idiopático e Secundário}

Os diversos quadros possíveis de autismo podem ser divididos em: Idiopáticos - sem causa definida - o qual compreende a maioria dos casos e se espera que diversos genes estejam associados, e secundários, onde agentes ambientais, anomalias cromossômicas ou alterações gênicas podem ser identificadas. Apenas $5-10 \%$ dos indivíduos com autismo se enquadram como secundário, os restantes 90 $95 \%$ possuem autismo idiopático (LINTAS; PERSICO, 2009).

Para TEA secundário, o modelo multifatorial de interferências na gestação parece ser o que melhor explica a etiologia destes casos. Estudos com melhor desenho metodológico apontam o incremento das idades materna e paterna, baixo peso ao nascimento e anóxia perinatal como os principalmente associados a TEA (SANCHES; BRUNONI, 2010). Doenças maternas como rubéola também se apresentam como fator importante (JOHNSON et al., 2007), além da exposição a xenobióticos durante a gestação. De acordo com Deth et al. (2008), metais pesados como arsênico, chumbo e mercúrio são conhecidos como as três substâncias mais perigosas pelo departamento de saúde humana dos Estados Unidos, apresentado consequências associadas a danos neurológicos, incluindo déficit de atenção, hiperatividade e baixo Ql, sintomas também presentes em autismo. Outros metais pesados como cádmio, manganês e níquel exercem efeitos similares e estão envolvidos com doenças neurodegenerativas, como Alzheimer e Mal de Parkinson.

$\mathrm{Ng}$ et al. (2017), elencaram diversos fatores de risco além do já conhecido acúmulo de metais pesados, como: produtos químicos consequentes de tráfego, idade parental avançada, nascimento prematuro, baixo peso ao nascer, hiperbilirubinemia e complicações na gravidez. Dentre os fatores químicos, os poluentes e metais pesados são de fato os maiores alvos de estudos de associação com o TEA Por sua vez a exposição a poluentes do ar pode resultar em doenças cardiovasculares, respiratórias e neurológicas, uma vez que desencadeiam inflamações e estresse oxidativo, com alguns sintomas similares aos observados em TEA. Já dentre os fatores fisiológicos, as características parentais e complicações na gravidez são os principais temas, onde é encontrada maior frequência de filhos com TEA quando os pais apresentam 40 e 49 anos - em comparação com pais de até 29 anos.

\section{Aspectos Epidemiológicos do TEA}

O TEA, apresenta prevalência de 1 a cada 68 crianças com até 8 anos, apresentando variação entre diferentes sexos e etnias. Aproximadamente 1 a cada 42 meninos encontram-se dentro do espectro autista, enquanto que para meninas o valor é de 1 a cada 189. Crianças de pele branca e não-hispânicas apresentam $30 \%$ a mais de chances de autismo do que crianças negras não-hispânicas e $50 \%$ a mais do que crianças hispânicas. Sobre os dados de Deficiência Intelectual (DI), 31\% dos indivíduos com TEA foram classificados com QI < 70, 23\% dos indivíduos com QI entre 71-85 e 46\% com QI >85. Enquanto que a média de diagnóstico para o TEA é de 53 meses (CENTERS FOR DISEASE CONTROL AND PREVENTION, 2014).

\section{Distribuição no Sexo}

O TEA apresenta prevalência no sexo masculino consideravelmente maior, com estudos como o de Frazier e Hardan (2016) classificando o sexo masculino como grande fator de risco para o distúrbio. A literatura aponta proporções de distribuição masculino/feminino variando de 2:1 até $6: 1$, sendo que em indivíduos com comprometimento cognitivo a proporção média é 1,3:1 e em indivíduos sem comprometimento cognitivo a proporção é de 7-10:1.

De acordo com Jacquemont et al. (2014), famílias com caso de autismo feminino apresentam risco mais elevado de novas ocorrências do que famílias com autismo masculino, podendo este dado ser expandido para a maioria das Desordens Neurodesenvolvimentais (DN). No estudo female protective model sobre um possível modelo que justificasse a menor prevalência de DN em mulheres, Jacquemont et al. (2014) concluem que a diferença entre os sexos é geneticamente demonstrada por um excesso de Copy-Number Variants (CNV's) deletérios três vezes maior em mulheres afetadas, além de um excesso de Single-Nucleotide Variants (SNV's) também deletérios e exclusivos em mulheres afetadas, sendo inclusive ambos transmitidos maternalmente.

Embora a divergência entre as taxas de distribuição por sexo causada por uma carga genética diferenciada encontre respaldo na literatura, Miltra et al. (2016) discorda da hipótese de carga genética tanto quanto de outras três hipóteses: contribuição do cromossomo X, interação gene-ambiente por cascatas hormonais e influência de genes expressados diferencialmente em cada sexo no desenvolvimento inicial do cérebro. Para Miltra et al. (2016) os mesmos mecanismos que agem na diferenciação sexual secundária influenciam alterando o risco para TEA durante o desenvolvimento, ou seja, uma pleiotropia entre características antropométricas e doenças complexas com diferenciação sexual, uma vez que foram encontradas fortes evidências de que aspectos dismórficos de características como altura, peso e medidas de cintura e quadril, contribuem desproporcionalmente para a associação com TEA. Em termos genéticos, há desequilíbrio - afetando particularmente mulheres - em SNPs, sendo observado que em mulheres com TEA SNPs envolvidos em características antropométricas apresentam enriquecimento do sinal de associação, o que não ocorre na mesma classe de SNPs comuns a ambos os sexos. 


\section{Bases Neurobiológicas do TEA}

O TEA é uma desordem que leva a efeitos no neuro-desenvolvimento, apresentando sintomas ainda mais expressivos na infância e que de acordo com Buck et al. (2014) se atenuam na vida adulta, embora muitas vezes não desapareçam. Skokauskas e Gallagher (2012) observaram que crianças com TEA frequentemente sofrem de alguma condição psiquiátrica. Buck et al. (2014), através do seu trabalho investigando 129 indivíduos autistas, concluíram que $56.6 \%$ dos participantes do estudo apresentaram alguma desordem psiquiátrica associada, sendo que $39,5 \%$ dos participantes sofriam de ansiedade e $9 \%$ de transtorno bipolar. De Bruin et al. (2007) relatam outras desordens psiquiátricas comuns, como desequilíbrio de humor, hiperatividade e déficit de atenção, além de transtorno desafiador opositivo, ocorrendo ao menos uma desordem por paciente em $80,9 \%$ das 94 crianças estudas em seu trabalho.

Guo et al. (2017) destacam uma redução na amplitude das baixas frequências cerebrais em indivíduos com TEA no precúneo direito e no giro occipital médio esquerdo durante todos os estágios de desenvolvimento, em outras palavras um padrão de desenvolvimento aberrante de atividade cerebral espontânea observados em casos de TEA com déficit social.

\section{Bases Genéticas do TEA}

De acordo com Bailey et al. (1995), o TEA é um transtorno genético com taxa de concordância - considerando especificamente o autismo - de $60 \%$ para gêmeos monozigóticos (MZ) e $0 \%$ para dizigóticos (DZ), enquanto que considerando todo o espectro, nomeadamente TEA, a taxa de concordância foi de $92 \%$ para gêmeos $\mathrm{MZ}$ e $10 \%$ para DZ.

Estudos como o de Hallmayer et al. (2011), apresentam taxas para TEA diferenciadas em relação as primeiras pesquisas - provavelmente por considerarem um número amostral maior - com $77 \%$ de concordância para gêmeos $M Z$ masculinos e $50 \%$ para gêmeos femininos $M Z$. Já as taxas de concordância para gêmeos DZ foram de $31 \%$ para o sexo masculino e $36 \%$ para o sexo feminino. Estes estudos observaram maior concordância dizigótica, levando a uma estimativa mais moderada da herdabilidade genética. De forma geral o risco de recorrência é de cerca de 10,1\% em uma família com uma criança autista (RISCH et al., 2014).

Atualmente o SFARI Gene Autdb, um banco de dados comunitário de considerável destaque e restrito a pesquisas com TEA, aponta 826 genes associados, e mais de 300 genes candidatos (SFARI, 2016). É interessante rever o progresso até os dias atuais, uma vez que em 1999, Risch et al. afirmaram "mesmo que seja amplamente aceito que não há um gene único para autismo, é difícil predizer o número de regiões genéticas, ou loci, que contribuem, estimando que aproximadamente 15 genes possam estar envolvidos". Já nos anos 2000 o valor estimado para o número de genes já girava por volta de 100 (GUPTA; STATE,
2006) com pesquisas vagamente afirmando "acredita-se que se trata de uma alteração envolvendo múltiplos genes e diversos cromossomos, para a manifestação do quadro observado em pessoas com autismo" (RAMOZ et al., 2004). Até o ano de 2011 o número de genes candidatos já era 171 (AUTISM DATASET, 2016).

Durante décadas diversos estudos, buscando genes candidatos, foram elaborados e assim regiões cromossômicas também foram descobertas. Muitos pesquisadores apontam os cromossomos 2 e 7 como os mais concentradores de genes envolvidos no autismo. A região 2q31.1 foi avaliada por IMGSAC (2001), encontrando ligações genéticas sugestivas dentro da mesma região. A porção 7q, por exemplo já foi amplamente estudada com trabalhos de Yonan et al. (2003) e Rutter (2005) que discutiram sobre ligação gênica neste cromossomo. Estudos em outras regiões, como as encontradas nos cromossomos 1, 5, 15 e 16, revelaram evidências de ligação gênica em certos intervalos. A região 15q11-13 é o sítio mais frequente de anormalidades cromossômicas detectados no TEA, de acordo com Gupta e State (2006), podendo ser encontrados pesquisas como a de Shao et al. (2003) focando apenas na região 15q11-13 e identificando associação entre ligações genéticas herdáveis entre gerações e o fenótipo. Outros trabalhos, como o de Yonan et al. (2003), mostraram resultados em pesquisas com a região $17 q$, estudando 345 famílias multiplex - aquelas com 2 ou mais casos entre parentes de 1 응 ou $2^{\circ}$ grau - e encontrando altos valores de linkages (ligações gênicas herdáveis entre gerações de indivíduos, associadas a um fenótipo).

A avaliação citogenética também se mostra presente em estudos de autismo. A revisão de Veenstra-VanderWeele, Christian e Cook (2004) aponta que em 1826 indivíduos autistas cariotipados, 78 apresentaram anormalidades. 0 estudo relata ainda que alterações cromossômicas foram encontradas em todos os 22 cromossomos autossômicos, no par sexual e ainda associadas a mutações dinâmicas, como SXF (VEENSTRA-VANDERWEELE; CHRISTIAN; COOK, 2004). É estimado que citogeneticamente sejam identificadas 6-7\% de alterações em pacientes com autismo (MARSHALL et al., 2008).

\section{Associação do TEA com Comorbidades: Autism Plus}

$A$ base genética do autismo, bem como a correlação deste com outras comorbidades, tem sido destacada em um número extenso de estudos. 0 trabalho de Gillberg (1998) demonstra a relação entre autismo e diversas anormalidades cromossômicas, sugerindo inclusive que em todos os cromossomos podem haver alterações relacionadas a condição autista. Castermans et al. (2004) através dos seus estudos sobre autismo apontaram que 5 a $10 \%$ dos casos ocorrem em comorbidade com outras desordens, como SXF, identificando assim a necessidade de maior investigação para cada caso autista. Do mesmo modo, Veenstra-VanderWeele, Christian e Cook (2004) relataram em seu trabalho a ocorrência de deleções da 
região 15q11-13 do cromossomo paterno em associação com Prader-Willi e a ocorrência de deleções na região 15q11-13 do cromossomo materno em associação com a Síndrome de Angelman.

O termo "Autism Plus", utilizado por Gillberg e Fernell (2014) fundamenta a associação entre autismo e comorbidades diversas. $\mathrm{O}$ trabalho aponta um real aumento de diagnóstico e registro da condição autista, e também de Autism Plus e objetivou chamar a atenção para os erros de diagnóstico, onde a condição associada não é adequadamente investigada e o paciente recebe o diagnóstico apenas de autista.

A Síndrome de Down (SD) é uma desordem genética causada pela presença de parte ou de todo um cromossomo 21 extra, sendo a causa genética geralmente não hereditária, mais comum de deficiência intelectual (Jl; CAPONE; KAUFMANN, 2011). A estimativa de associação da SD com TEA é de $5 \%$ a 39\% (REILLY, 2009; DIGUISEPPI et al., 2010) enquanto que a estimativa de associação entre SD e especificamente autismo - enquanto o autismo ainda era considerado como subclasse de TEA - varia entre $1 \%$ a 12\% (REILLY, 2009).

Outras síndromes de microdeleções, como a Síndrome de Rubinstein-Taybi (SRT), também apresentam registros da co-ocorrência com autismo. A SRT, por exemplo, é causada por mutações e deleções nos genes da região 16 13.3 ou por mutação nas proteínas E1A-binding ou CBP (WAITE et al., 2015). Quando a comorbidade é confirmada, alguns sinais autísticos evidentes são observados, como o comportamento repetitivo, o que de acordo com Turner (1999) indica a associação entre SRT e autismo, com dados de Stevens, Pouncey e Knowles (2011) relatando que $62 \%$ dos indivíduos com SRT apresentavam comportamentos autísticos repetitivos.

A Síndrome do X-Frágil (SXF) é a causa hereditária mais comum de deficiência intelectual, afetando 1 a cada 2500 indivíduos (HAGERMAN, 2008). O fenótipo mais comum inclui distúrbios cognitivos e alterações comportamentais em graus variados, além de anomalias físicas, como orelhas grandes, face longa, macrocefalia e macroorquidismo (HAGERMAN, 2002). A SXF é causada por uma mutação instável no gene Fragile $X$ Mental Retardation 1 (FMR1), onde uma expansão de trinucleotídeos CGG no gene leva a um silenciamento e consequentemente a não transcrição do gene na proteína FMR1. A associação entre autismo e SXF foi primeiro reportada em 1982, por Brown et al. (1982) sendo a associação mais comum entre os indivíduos com SXF.

A ocorrência de autismo com a Síndrome do X-Frágil está associada com a linguagem e deficiência social, além de baixo QI comparado com crianças com SXF apenas, como concluiu o estudo com 90 indivíduos com SXF, e Autism Plus SXF, de Gárcia-Nonell et al. (2008). Klusek, Martin e Losh (2014) descrevem ainda em seu trabalho com 35 mulheres e 51 homens com SXF, que $25 \%$ do total de pacientes apresentou comorbidade entre SXF e TEA, relatando que os valores para homens com a comorbi- dade foram três vezes superiores ao encontrado para as mulheres ( $75 \%$ vs. $25 \%$ ).

\section{CONCLUSÃO}

Os dados deste trabalho demonstram a importância de fatores genéticos na manifestação do Transtorno de Espectro Autista (TEA). A variabilidade fenotípica da condição é relacionada à existência de número superior a 800 genes associados, sendo destes mais de 300 descritos como genes candidatos, e à influência de fatores ambientais no desenvolvimento.

A associação conhecida como Autism Plus explica a comorbidade do autismo com algumas síndromes de etiologia genética. Estudos de bioinformática indicam a existência de segmentos cromossômicos preferencialmente envolvidos no Autismo Plus, a exemplo dos cromossomos 15, 21, 22 e X. Estudos adicionais a respeito do Autism Plus poderão esclarecer o efeito pleiotrópico de genes nestes segmentos que além de promoverem uma condição sindrômica, como a Síndrome de Down pode estar envolvida na etiologia do TEA.

Novas pesquisas sobre Autism Plus, principalmente com a utilização de instrumentos da genômica, são recomendadas para a elucidação de fatores genéticos causais, tendo em vista o tratamento, prognóstico e prevenção desta condição ainda pouco conhecida, não obstante a sua ocorrência relativamente frequente.

\section{REFERÊNCIAS}

AMERICAN PSYCHIATRIC ASSOCIATION (APA). Diagnostic and statistical manual of mental disorders: DSM-IV.4 ed. Washington: APA, 1994.

AMERICAN PSYCHIATRIC ASSOCIATION (APA). Diagnostic and statistical manual of mental disorders: DSM-V. 5 ed. Washington: APA, 2013.

ASSUMPÇÃO, F. B.; PIMENTEL, A. C. M. Autismo infantil. Rev. Bras. Psiquiatr. São Paulo, v. 22, n. 1, p. 37-39, 2000.

AUTISM DATASET. Center of Bioinformatics, Peking University. Disponível em: <http://autismkb.cbi.pku.edu.cn/core_dataset.php>. Acesso em: 03 Oct. 2016.

BAILEY, A. et al. Autism as a strongly genetic disorder: evidence from a British twin study. Psychol. Med. New York, v. 25, n. 1, p. 63-77, 1995.

BAKER, J. P. Autism at 70: redrawing the bundaires. N. Engl. j. med. Boston, v. 369, n. 12, p. 1089-1091, 2013.

BROWN, W. T. et al. Association of fragile $X$ with autism. Lancet. London, v. 1, n. 8263 , p. 100, 1982.

BUCK, T. R. et al. Psychiatric Comorbidity and Medication Use in Adults with Autism Spectrum Disorder. J. Autism Dev. Disord. New York, v. 44, n. 12, p. 3063-3071, 2014.

CARVALHEIRA, G.; VERGANI, N.; BRUNONI, D. Genetics of autism. Rev. Bras. Psiquiatr. São Paulo, v. 26, n. 4, p. 270-272, 2004.

CASTERMANS, D. et al. Chromosomal anomalies in individuals with autism. Autism, London, v. 8, n. 2, p. 141-161, 2004.

CENTERS FOR DISEASE CONTROL AND PREVENTION. Prevalence of Autism Spectrum Disorder Among Children Aged 8 Years - Autism and Developmental Disabilities Monitoring Network, 11 Sites, United 
States. Morb. Mortal. Wkly Rep., Washington, v. 63, n. 2, p. 1-23, 2014.

DE BRUIN, E. I. et al. High rates of psychiatric co-morbidity in PDD-NOS. J. Autism. Dev. Disord. New York, v. 37, n. 5, p. 877-886, 2007.

DETH, R. et al. How environmental and genetic factors combine to cause autism: A redox/methylation hypothesis. Neurotoxicology, Amsterdam, v. 29, n. 1 , p. $190-201,2008$

DIGUISEPPI, C. et al. Screening for autism spectrum disorders in children with Down syndrome: population prevalence and screening test characteristics. J. Dev. Behav. Pediatr. Baltimore, v. 31, n. 3, p. 181-191, 2010.

FRAZIER, T. W.; HARDAN, A. Y. Equivalence of symptom dimensions in females and males with autism. Autism, London, p. 1-11, 2016.

GARCÍA-NONELL, C. et al. Secondary Medical Diagnosis in Fragile $X$ Syndrome With and Without Autism Spectrum Disorder. Am. J. Med. Genet., New York, v. 0, n. 15, p. 1911-1916, 2008.

GILLBERG, C. Chromosomal Disorders and Autism. J. Autism. Dev. Disord. New York, v. 28, n. 5, p. 415-425, 1998.

GILLBERG, C.; FERNELL, E. Autism plus versus autism pure. J. Autism. Dev. Disord. New York, v. 44, n. 12, p. 3274-3276, 2014.

GUO, X. et al. Atypical developmental trajectory of local spontaneous brain activity in autism spectrum disorder. Sci. Rep., London, v. 7, n. 39822, 2017.

GUPTA, A. R.; STATE, M. W. Autismo: genética. Rev. Bras. Psiquiatr. São Paulo, v. 28, n. 1, p. 29-38, 2006.

HAGERMAN, P. J. The fragile X prevalence paradox. Am. J. Med. Genet., New York, v. 45, n. 8, p. 498-499, 2008.

HAGERMAN, R. J. Physical and behavioral phenotype. In: (Org.) Fragile $X$ syndrome: diagnosis, treatment and research. 3 ed. Baltimore: The Johns Hopkins University Press. 2002. p.103-109.

HALLMAYER, J. et al. Genetic Heritability and Shared Environmental Factors Among Twin Pairs With Autism. Arch. Gen. Psychiatr. Chicago, v. 68, n. 11, p. 1095-1102, 2011.

INTERNATIONAL MOLECULAR GENETIC STUDY OF AUTISM CONSORTIUM (IMGSAC). A genomewide screen for autism: strong evidence for linkage to chromosomes 2q, 7q, and 16p. Am. J. Med. Genet. New York, v. 69, n. 3, p. 570-581, 2001.

JACQUEMONT, S. et al. A Higher Mutational Burden in Females Supports a "Female Protective Model" in Neurodevelopmental Disorders. Am. J. Hum. Genet., Chicago, v. 94, n. 3, p. 415-425, 2014.

JI, N. Y.; CAPONE, G. T.; KAUFMANN, W. E. Autism spectrum disorder in Down syndrome: cluster analyis of Aberrant Behaviour Checklist data supports diagnosis. J. Intellect. Dis. Res. London, v. 55, n. 11, p. 1064-1077, 2011.

JOHNSON, C. P; MYERS, S. M. Concil on Children With Disabilities: identification and Evaluation of children with autism spectrum disorders. Pediatrics, Evanston, v. 120, n. 5, p. 1183-1215, 2007.

KLUSEK, J.; MARTIN, G. E.; LOSH, M. Consistency between research and clinical diagnoses of autism among boys and girls with fragile $X$ syndrome. J. Intellect. Dis. Res. London, v. 58, n, 10, p. 940-952, 2014.
LINTAS, C.; PERSICO, A. M. Autistic phenotypes and genetic testing: state-of-the-art for the clinical geneticist. Am. J. Med. Genet. New York, v. 46, n. 1, p. 1-8, 2009.

MARSHALL, C. R. et al. Structural Variation of Chromosomes in Autism Spectrum Disorder. Am. J. Hum. Genet. Chicago, v. 82, n. 2, p. 477-488, 2008.

MILTRA, I. et al. Pleiotropic Mechanisms Indicated for Sex Differences in Autism. PLoS Genet. San Francisco, v. 12, n. 11, p. 2-27, 2016.

NG, M. et al. Environmental factors associated with autism spectrum disorder: a scoping review for the years 2003-2013. Health Promot. Chronic. Dis. Prev. Can., Canadá, v. 37, n. 1, p. 1-23, 2017.

RAMOZ, N. et al. Linkage and association of the mitochondrial aspartate/glutamate carrier SLC25A12 gene with autism. Am. J. Psychiatr. Arlington, v. 161, n. 4, p. 662-669, 2004.

REILLY, C. Autism spectrum disorders in Down syndrome: a review. Res. Autism. Spectr. Disord., London, v. 3, n. 4, p. 829-839, 2009.

$\mathrm{RISCH}, \mathrm{N}$. et al. A genomic screen of autism: evidence for a multilocus etiology. Am. J. Hum. Genet., Chicago, v. 65, n. 2, p. 493-507, 1999.

$\mathrm{RISCH}, \mathrm{N}$. et al. Familial Recurrence of Autism Spectrum Disorder: Evaluating Genetic and Environmental Contributions. Am. J. Psychiatr. Arlington, v. 171, n. 11, p. 1206-1213, 2014.

RUTTER, M. Genetic influences and autism. IN: VOLKMAR, F. R.; PAUL, R.; KLIN, A.; COHEN, D. (Org.). Handbook of Autism and Pervasive Developmental Disorders. 3 ed. New Jersey: John Wiley and Sons, 2005. v. 1. p. $425-452$.

SANCHES, C. P.; BRUNONI, D. Intercorrências perinatais em indivíduos com transtornos Invasivos do desenvolvimento: uma revisão. Cadernos de Pós-Graduação em Distúrbios do Desenvolvimento, São Paulo, v. 10, n. 1, p. 21-31, 2010.

SHAO, Y. et al. Fine mapping of autistic disorder to chromosome 15q11-q13 by use of phenotypic subtypes. Am. J. Hum. Genet. Chicago, v. 72, n. 3, p. 39-48, 2003.

SKOKAUSKAS, N.; GALLAGHER, L. Mental health aspects of autistic spectrum disorders in children. J. Intellect. Dis. Res. London, v. 56, n. 3, p. 248-257, 2012.

STEVENS, C. A.; POUNCEY, J.; KNOWLES, D. Adults With Rubinstein-Taybi Syndrome. Am. J. Med. Genet. New York, v. 155A, n. 7, p.1680-1684, 2011.

TURNER, M. Annotation: Repetitive Behaviour in Autism: A Review of Psychological Research. J. Child Psychol. Psychiatry Allied Discipl. Elmsford, v. 40, n. 6, p. 839-849, 1999.

VEENSTRA-VANDERWEELE, J.; CHRISTIAN, S. L.; COOK, E. H. Autism as a paradigmatic complex genetic disorder. Annu. Rev. Genomics Hum. Genet. Palo Alto, v. 5, p. 379-405, 2004.

WAITE, J. et al. Repetitive Behavior in Rubinstein-Taybi Syndrome: Parallels with Autism Spectrum Phenomenology. Res. Autism. Spectr. Disord. London, v. 45, n. 5, p. 1238-1253, 2015.

YONAN, A. L. et al. A genomewide screen of 345 families for autismsusceptibility loci. Am. J. Med. Genet. New York, v. 73, n. 4, p. 886-897, 2003
Submetido em: 27/03/2017

Aceito em: 19/06/2017 